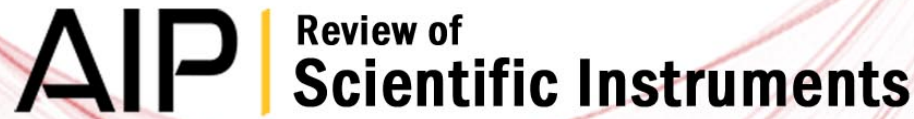

Sensitive thermal transitions of nanoscale polymer samples using the bimetallic effect: Application to ultra-thin polythiophene

O. Ahumada, M. M. Pérez-Madrigal, J. Ramirez, D. Curcó, C. Esteves et al.

Citation: Rev. Sci. Instrum. 84, 053904 (2013); doi: 10.1063/1.4804395

View online: http://dx.doi.org/10.1063/1.4804395

View Table of Contents: http://rsi.aip.org/resource/1/RSINAK/v84/i5

Published by the AIP Publishing LLC.

Additional information on Rev. Sci. Instrum.

Journal Homepage: http://rsi.aip.org

Journal Information: http://rsi.aip.org/about/about_the_journal

Top downloads: http://rsi.aip.org/features/most_downloaded

Information for Authors: http://rsi.aip.org/authors

\section{ADVERTISEMENT}

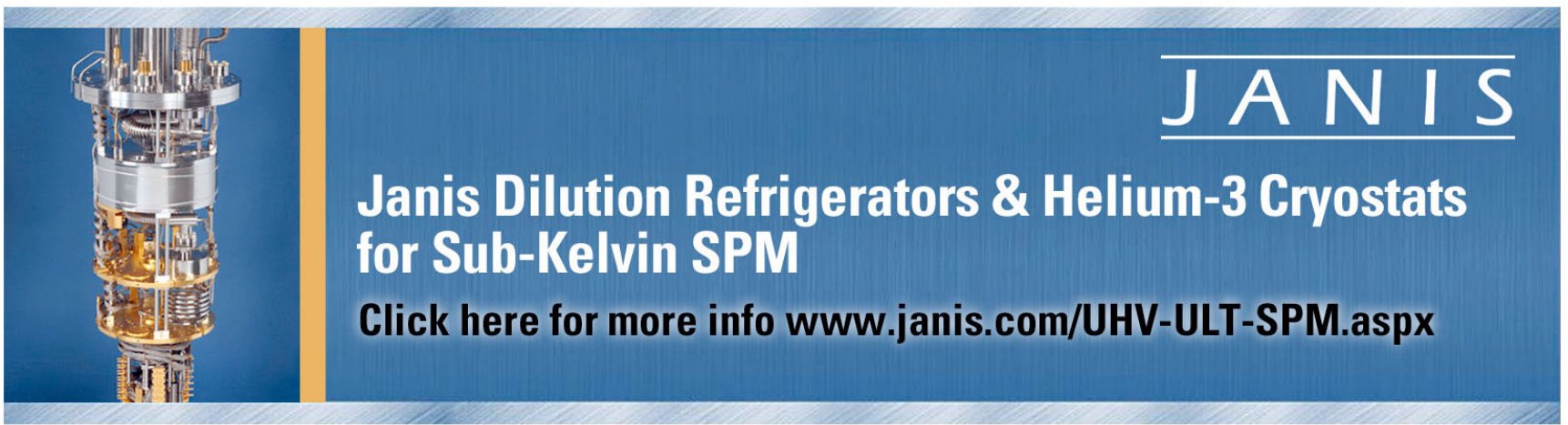




\title{
Sensitive thermal transitions of nanoscale polymer samples using the bimetallic effect: Application to ultra-thin polythiophene
}

\author{
O. Ahumada, ${ }^{1, \text { a) }}$ M. M. Pérez-Madrigal, ${ }^{2,3}$ J. Ramirez, ${ }^{1,4}$ D. Curcó, ${ }^{5}$ C. Esteves, ${ }^{1}$ \\ A. Salvador-Matar, ${ }^{1}$ G. Luongo, ${ }^{1}$ E. Armelin, ${ }^{2,3}$ J. Puiggalí, ${ }^{2,3}$ and C. Alemán ${ }^{2,3, a)}$ \\ ${ }^{1}$ Mecwins S.L., Parque Científico de Madrid PTM, C/Santiago Grisolía 2, Tres Cantos, Madrid E-28760, Spain \\ ${ }^{2}$ Departament d'Enginyeria Química, E.T.S d'Enginyers Industrials de Barcelona, \\ Universitat Politècnica de Catalunya, Av. Diagonal 647, Barcelona E-08028, Spain \\ ${ }^{3}$ Center for Research in Nano-Engineering, Universitat Politècnica de Catalunya, Campus Sud, Edifici C', \\ C/Pasqual $i$ Vila s/n, Barcelona E-08028, Spain \\ ${ }^{4}$ Departamento de Ingeniería Qúmica Industrial y Medio Ambiente, Escuela Superior de Ingenieros \\ Industriales Univerdidad Politécnica de Madrid, Jose Gutierrez Abascal, 2 Madrid E28006, Spain \\ ${ }^{5}$ Departament d'Enginyeria Química, Facultat de Química, Universitat de Barcelona, Martí i Franques 1, \\ Barcelona E-08028, Spain
}

(Received 29 November 2012; accepted 24 April 2013; published online 15 May 2013)

\begin{abstract}
A sensitive nanocalorimetric technology based on microcantilever sensors is presented. The technology, which combines very short response times with very small sample consumption, uses the bimetallic effect to detect thermal transitions. Specifically, abrupt variations in the Young's modulus and the thermal expansion coefficient produced by temperature changes have been employed to detect thermodynamic transitions. The technology has been used to determine the glass transition of poly(3-thiophene methyl acetate), a soluble semiconducting polymer with different nanotechnological applications. The glass transition temperature determined using microcantilevers coated with ultra-thin films of mass $=10^{-13} \mathrm{~g}$ is $5.2^{\circ} \mathrm{C}$ higher than that obtained using a conventional differential scanning calorimeter for bulk powder samples of mass $=5 \times 10^{-3} \mathrm{~g}$. Atomistic molecular dynamics simulations on models that represent the bulk powder and the ultra-thin films have been carried out to provide understanding and rationalization of this feature. Simulations indicate that the film-air interface plays a crucial role in films with very small thickness, affecting both the organization of the molecular chains and the response of the molecules against the temperature. (C) 2013 AIP Publishing LLC. [http://dx.doi.org/10.1063/1.4804395]
\end{abstract}

\section{INTRODUCTION}

Miniaturized calorimeters (i.e., nanocalorimeters), which are predominantly based on silicon chips, are designed to perform nanoscale thermal analysis with high sensitivity on extremely small amounts of sample (nanograms, picoliters) under very fast operating rates. ${ }^{1-3}$ Reduced sample size means reduced sample consumption, which demands higher resolution and better thermal isolation. Temperature can be measured using integrated thermocouples or by determining changes in the temperature coefficient of resistance of the heater element. ${ }^{4}$ An optical technique has been recently developed to calibrate the thermal response of the chips, avoiding errors in the extrapolation from low to high temperatures. ${ }^{5}$ Although the use of nanocalorimetry started in the 1970 s by determining the heat capacity of very thin films, ${ }^{6}$ essential progress in both development and applications has been made in the last decade. Therefore, nanocalorimetry is still considered as an emerging field. Recently, nanocalorimetry has been applied to a variety of materials including metal, ceramics, polymers, and biomolecules. ${ }^{4,7,8}$ Thus, nanocalorimeters have been adapted to carry out unusual small-scale measures, for example, interfacial reactions, ${ }^{9,}{ }^{10}$ phase transitions, ${ }^{7}$ nanoscale biologi-

a) Authors to whom correspondence should be addressed. Electronic addresses: oahumada@mecwins.com and carlos.aleman@upc.edu cal reactions, ${ }^{2}$ folding-unfolding processes in proteins,,${ }^{11}$ and absorption phenomena in ultra-thin films. ${ }^{12}$

Sensitive calorimeters based on the cantilever technology represent an alternative to the chip-based nanocalorimeters. Thus, the cantilever deflection resulting from the absorption of heat, either at a localized spot on the cantilever or along its entire length, can be modeled to determine thermal transitions. The small size and heat capacity of micro-cantilevers make them remarkable nanocalorimeters with picojoule sensitivities and milliseconds time resolution. ${ }^{13}$ This represents a significant improvement with respect to conventional differential scanning calorimeters (DSCs) and infrared detection techniques, which typically show sensitivities of $0.2 \mathrm{~mJ}$ and $6 \mathrm{~nJ}$, respectively, and time resolutions on the order of the second. ${ }^{14}$ For example, cantilever-based nanocalorimetry has been used to measure enthalpy changes in picoliter volumes of solid samples during phase transitions of $n$-alkanes, ${ }^{13}$ to detect molecules adsorbed on the layer coating of the cantilever when it is catalytically active, ${ }^{15}$ and to determine the thermomechanical properties of polymeric samples in the vicinity of their glass transition temperature $\left(\mathrm{T}_{\mathrm{g}}\right) .{ }^{16}$ Regarding the latter, Jung et al. ${ }^{16}$ used the variations in the resonance frequency and deflection of the cantilever with the temperature to determine the change in the effective modulus and volume of polystyrene when the temperature was close to the $T_{g}$.

In this work, we present a technology developed to determine thermal transitions in particular the $T_{g}$ of 
ultra-thin polymeric samples with a mass scale lower than the picogram. This promising technology named SCALA CAL, which opens the door to measure thermal transitions at the molecular level, represents a significant improvement in relation to currently available nanocalorimetric techniques. The work has been organized as follows. In Sec. II, the technology is described after providing a brief theoretical background. After this, the technology is evaluated at the proof concept level using nylon 6. Finally, in Sec. IV the new technology has been used to evaluate the $T_{g}$ of a soluble semiconducting polymer with important applications in nanotechnology. Specifically, Sec. IV has been distributed in three parts. The first provides a description of the experimental methods, the second compares the glass transition determined in samples with a mass of $\sim 10^{-13} \mathrm{~g}$ with that obtained from samples with $\sim 10^{-3} \mathrm{~g}$, and the last part shows a comprehensive Molecular Dynamics (MD) simulation study to rationalize the experimental observations at the molecular level.

\section{THEORETICAL BACKGROUND AND TECHNOLOGY}

Cantilever-based sensing technologies are emerging platforms with the advantage of being relatively cheap to massproduce. Micro-fabrication technologies have facilitated not only the design of micro-cantilevers optimized for various types of sensing applications but also the manufacturing of large arrays of cantilevers, making it possible to use various sensors in parallel. These cantilever-based sensors have been proved to be quite competitive with current sensing technologies because of their high sensitivity, fast response time, and small size (i.e., from $\mu \mathrm{m}$ to $\mathrm{nm}$ ). ${ }^{17-20}$

SCALA is the acronym for "Scanning Laser Analyzer," a recently developed technology for the optical read-out of cantilevers. $^{21,22}$ The aim of this work consists of the application of this technique, which allows the user to readout the profiles of microcantilever arrays using a picometric massscale and a processing speed of about ten cantilevers per second, on the analysis of the thermal properties of ultra-thin polymeric samples.

The application has end up with a new technology based on SCALA named SCALA CAL. The improvement in the scanning laser analyzer consists in the implementation of a calorimetric chamber containing a thermoelectric cooler and a cartridge heater. These elements are capable of sweeping the chamber's temperature from -50 up to $220^{\circ} \mathrm{C}$ by flowing thermostatized $\mathrm{N}_{2}$ to the sample. A holder, with multiple thermometer probes, is placed inside the chamber for a better mapping of the temperature. All the measurements are performed in nitrogen environment to avoid the oxidation of the sample. The temperature control is regulated by software realized in a LabVIEW environment, which allows to acquire data and to send commands to hardware.

The readout technique combines the optical beam deflection method and the automated two-dimensional scanning of a single laser beam by voice-coil actuators. Figure 1 shows a schematic representation of the experimental setup. A $1 \mathrm{~mW}$ laser diode is mounted on two perpendicular linear voice coil actuators, which allow two-dimensional non-hysteretic displacement over a range of several millimeters at speeds up to

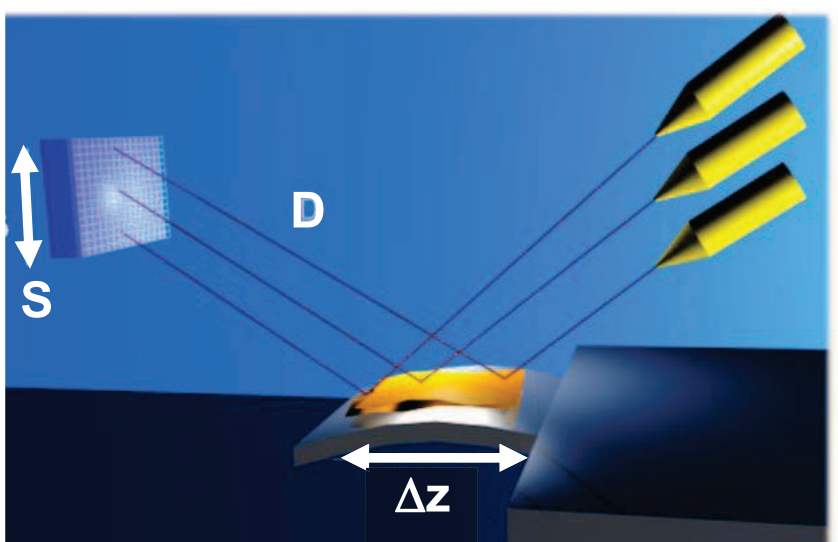

FIG. 1. Schematic representation of the experimental setup used to determine thermal properties.

$10 \mathrm{~mm} / \mathrm{s}$ and with an accuracy of $100 \mathrm{~nm}$. One of the scanning axes is oriented parallel to the cantilever longitudinal axis. The cantilever array is illuminated by the laser beam and a two-dimensional linear position detector (PSD) is arranged to collect the reflected beams. A convergent lens is used to decrease the spot size of the laser on the cantilevers to 5-10 $\mu \mathrm{m}$, approximately.

Location and characterization of cantilever sensors is carried out using TRACKER, an algorithm based on the recognition of reflected intensity patterns. This capability of SCALA allows the user to characterize a single sensor or an array of them in a fully automated process. Moreover, the algorithm is able to recognize both commercial and self-made mechanical sensors.

A change in the local slope at the cantilever surface results into a displacement of the reflected laser spot on the PSD, which is denoted $S$ (Figure 1). Thus, the displacement recorded by the PSD during the scan of the cantilever is described by

$$
S(x) \cong 2 D \frac{d z}{d x}(x)+x \cos \beta,
$$

where $z(x)$ is the cantilever profile along its longitudinal axis, $D$ is the distance between the cantilever and the PSD, and $\beta$ is the angle between the incident laser beam and the cantilever normal at its rest position. The second summand in Eq. (1) accounts for the effect of the laser beam displacement, which is generally small compared to the first summand associated to the slope variations. The cantilever profiles are obtained by integrating Eq. (1)

$$
z(x)=\frac{1}{2 D} \int_{0}^{x} S\left(x^{\prime}\right) d x^{\prime}-\frac{1}{4} \frac{x^{2}}{D} \cos \beta .
$$

This technology is attracting a growing interest due to their capabilities for high sensitivity, label-free detection, and small sample consumption. ${ }^{23,24}$ These properties are inherent in the nature of the nanomechanical response and the tiny size of the microcantilevers, with areas of $\sim 1000 \mu \mathrm{m}^{2}$. In addition, these sensors are scalable as the microfabrication techniques admit routine fabrication of arrays with tenths of 
cantilevers, which is very convenient for platforms devoted to complex chemical and biological analyses.

Cantilevers can be used as precise thermometers or calorimeters by exploiting the bimetallic effect. ${ }^{25}$ This phenomenon refers to the measurable bending produced by temperature changes in bimaterial systems, like a substrate covered on one side by another material coating. ${ }^{26,27}$ Similarly, coated microcantilevers undergo a deflection (i.e., differential stress) as a result of temperature changes due to the thermal expansion coefficient difference between the coating material of the cantilever and the cantilever itself. ${ }^{28}$ The deflection of a rectangular cantilever, $\Delta z$, produced by a change in temperature, $\Delta \mathrm{T}$, is expressed as ${ }^{29}$

$$
\Delta z=\frac{3}{t_{c}^{2} K}\left(\alpha_{b}-\alpha_{c}\right)\left(t_{b}+t_{c}\right) \ell^{2} \Delta T,
$$

where $\ell$ is the rectangular cantilever's length, $\alpha_{b} / \alpha_{c}$ and $t_{b} / t_{c}$ are the thermal expansion coefficient and the thicknesses of the cantilever beam/cantilever's coating, respectively. $K$ is expressed as

$$
K=4+6\left(\frac{t_{b}}{t_{c}}\right)+4\left(\frac{t_{b}}{t_{c}}\right)^{2}+\frac{E_{b}}{E_{c}}\left(\frac{t_{b}}{t_{c}}\right)^{3}+\frac{E_{c}}{E_{b}}\left(\frac{t_{c}}{t_{b}}\right),
$$

where $E_{b}$ and $E_{c}$ are the Young's modulus of the cantilever and of the coating, respectively.

The application of micromechanical sensors to thermal phase transition analyses of phase transitions is based on its compact size combined with very high sensitivity and short response times. The sensitivity of a bimaterial micromechanical sensor is $\sim 1 \mathrm{pJ}$ while the response time is $\sim 1 \mathrm{~ms} .{ }^{30}$ Accordingly, the limit of this application corresponds to enthalpy changes within attomolar quantities of material.

When a polymeric coating is heated around a thermodynamic transition (i.e., the temperature of the film is close to the $T_{g}$ or the melting temperature, $T_{m}$ ), parameters such as the Young's modulus and the thermal expansion coefficients undergo abrupt changes. The effects produced by such changes on the mechanical deflection of the cantilever are recognized as the thermodynamic transitions fingerprints using Eq. (4).

\section{PROOF OF CONCEPT: NYLON 6}

The effect of changes in the Young's modulus and the thermal expansion coefficient on the deflection of the cantilever has been evaluated using Eq. (3). Figure 2 represents the deflection curve calculated using the technical data sheet of commercial nylon 6 from DSM Engineering Plastics (PA6, Aukulon F-9183). The $\mathrm{T}_{\mathrm{g}}$ and $\mathrm{T}_{\mathrm{m}}$ of nylon 6 are visualized as changes in the slope at 57 and $217^{\circ} \mathrm{C}$, respectively. In region I of the curve, the cantilever bends linearly due to the fact that the thermal expansion coefficient of the polymer and the silicon are different. The slope of the deflection curve changes at the glass transition temperature (region II), which combines pronounced jump of the thermal expansion coefficient and a decay of the Young's modulus. Finally, the Young's modulus practically vanishes at the melting temperature (region III), producing another drastic change in the deflection. The $T_{g}$ and $\mathrm{T}_{\mathrm{m}}$ values obtained from the deflection curve are in good

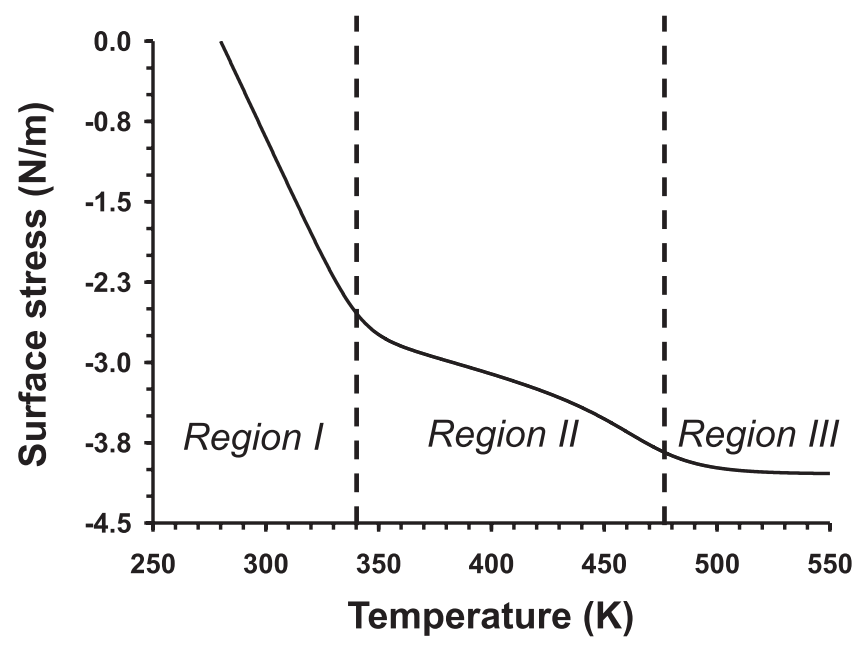

FIG. 2. Calculation of the deflection curve against the temperature (Eq. (3)) for commercial nylon 6 .

agreement with those reported in the technical data sheet of Aukulon F-9183 (67 and $207^{\circ} \mathrm{C}$, respectively), which were determined from the bulk using conventional DSC methods.

\section{APPLICATION TO A SOLUBLE POLYTHIOPHENE}

The technology described above has been applied to poly(3-thiophene methyl acetate) (P3TMA, Scheme 1), a soluble polythiophene derivative. The choice of this semiconducting material is based on its recently reported nanotechnological application. Thus, P3TMA was combined with a biodegrable polyester to prepare semiconducting nanomembranes (i.e., thickness from 20 to $80 \mathrm{~nm}$ ) that were used as bioactive platforms for tissue engineering. ${ }^{31,32}$ Furthermore, P3TMA dispersed as nanoparticles was found to be a powerful anticorrosive additive of organic coating, showing better protecting abilities than zinc phosphate compounds. ${ }^{33}$ As a result, this material was patented for its application as anticorrosive pigment to low volatile organic compounds (VOC) solvent-based epoxy and alkyd formulations. ${ }^{34,35}$ Accordingly, comparison of the thermal properties between bulk P3TMA powder and samples in which the P3TMA chains are organized in nanometric length-scales deserve consideration.

\section{A. Experimental and computational methods}

P3TMA was synthesized by oxidative coupling following the procedure described by Kim et al. ${ }^{36}$ After removal of residual oxidant and oligomers, P3TMA was a power soluble in different organic and polar solvents at room $\left(25^{\circ} \mathrm{C}\right)$ and high temperature $\left(60^{\circ} \mathrm{C}\right) .{ }^{37}$

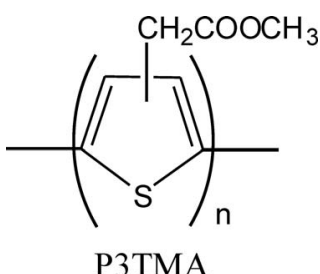

SCHEME 1. 
Conventional DSC measurements were obtained using a TA Instruments Q100 series equipped with a refrigerated cooling system (RCS) operating at temperatures between $-90^{\circ} \mathrm{C}$ and $600{ }^{\circ} \mathrm{C}$. Experiments were conducted under a flow of dry nitrogen with a sample weight of approximately $5 \mathrm{mg}$, calibration being performed with indium. Heating runs were performed at different temperatures, ranging from 1 to $20^{\circ} \mathrm{C} / \mathrm{min}$, with samples previously quenched from the melt state by cooling at the maximum rate allowed by the equipment. Determination of the $\mathrm{T}_{\mathrm{g}}$ was carried out with the TAUniversal Analysis software furnished with the instrument.

Samples for nanocalorimetric measurements were prepared by spin-coating a tetrahydrofurane (THF) solution of P3TMA onto previously cleaned microcantilevers. In order to achieve the maximum affinity between the polymer and the surface of the substrate, different cleaning procedures and spinning conditions (i.e., concentration of the solution, spincoater speed and time, and temperature) were tested using silicon squares obtained from silicon wafer as substrate. The main characteristics of this silicon substrate, which is cheaper than cantilevers, are similar to those of the chips (i.e., 99.9\% $\mathrm{Si}$ ), resulting very appropriate for all trials. Four different cleaning procedures were tested: (i) immersion in a piranha solution (i.e., a mixture of sulfuric acid and hydrogen peroxide) for $30 \mathrm{~min}$; (ii) immersion in water mixed with detergent from Procter \& Gamble for $30 \mathrm{~min}$; (iii) exposure to UV ozone cleaner from Novascan; and (iv) immersion in acetone, a heavy duty degreaser, and then into isopropanol, 15 min in each solvent. Optimal microscopy analysis indicated that (ii) produced the most homogeneous and continuous films. The cleaning action of the detergent, which acts as surfactant in conjunction with water, should be attributed to the micelles that surround grease particles causing them to disperse in water. Regarding the spinning conditions, they were optimized to get a thickness lower than $100 \mathrm{~nm}$ at both the center and border regions of the microcantilevers. After trial experiments, cantilevers for calorimetric measurements were coated at room temperature applying a spin coater speed of $1200 \mathrm{rpm}$ for $60 \mathrm{~s}$ to a $5 \mathrm{mg} / \mathrm{ml}$ P3TMA solution. Accordingly, SCALA CAL has been used for thermal characterization of P3TMA, which was deposited using such spinning conditions on commercial arrays of 8 cantilevers from Concentris $\mathrm{GmbH}$ cleaned with a mixture of water and detergent. Nine chips, which represent a total of 72 different sensors, were measured. The rate of heating was $1^{\circ} \mathrm{C} / \mathrm{min}$. Together with every functionalized sensor, a control (i.e., a cantilever where no polymer has been deposited) was measured.

Optical microscopy observations were performed using a Zeiss Axioskop 40 Pol light polarizing microscope equipped with a Linkam temperature control system configured by a THMS 60. Micrographs were taken with a Zeiss Axios MRC digital camera. Scanning electron microscopy (SEM) studies were carried out using a Focused Ion Beam Zeiss Neon40 scanning electron microscope equipped with an energy dispersive X-ray (EDX) spectroscopy system operating at $3.0 \mathrm{kV}$. Samples were mounted on a double-sided adhesive carbon disc and sputter-coated with a thin layer of carbon to prevent sample charging problems. Atomic force microscopy (AFM) images were obtained with a Molecular Imaging
PicoSPM using a NanoScope IV controller under ambient conditions. The tapping mode AFM was operated at constant deflection (i.e., vertical constant force with triangular shaped gold-coated silicon nitride). RMS roughness was determined using the statistics application and tools of the NanoScope Analysis software version 1.20. The scan window size was 5 $\times 5 \mu \mathrm{m}^{2}$. The row scanning frequency was set to $1 \mathrm{~Hz}$ and the physical tip-sample motion speed was $10 \mathrm{~mm} / \mathrm{s}$.

MD simulations were carried out with the NAMD program ${ }^{38}$ using a molecular system constituted by 40 identical chains, each one involving 15 repeating units with headto-tail polymer linkages and an alternated disposition (i.e., anti conformation). Periodic boundary conditions using the nearest image convention were applied in the three (i.e., $x$-, $y$-, and $z$-edges of the simulation box) and two directions (i.e., $x$ - and $y$-edges of the simulation box) to represent the bulk powder (BP model) and the ultra-thin coating (UTC model) samples, respectively. The potential energy was computed using the AMBER force-field, ${ }^{39}$ all the bonding and van der Waals parameters required for the DPs under study being taken from Generalized AMBER force-field (GAFF). ${ }^{40}$ Atomic charges were adjusted using the Restrained ElectroStatic Potential (RESP) strategy. ${ }^{41}$ Atom-pair distance cutoffs were applied at $12 \AA$ to compute van der Waals and electrostatic interactions. Bond lengths involving hydrogen atoms were constrained using the SHAKE algorithm with a numerical integration step of $2 \mathrm{fs}^{42}$

Initial microstructures for the BP and UTC models were constructed using a recently developed computational tool, which was developed to represent the packing of polythiophene derivatives. ${ }^{43}$ In order to identify the changes induced by the glass transition at the microscopic level, each system was simulated at three temperatures, $\mathrm{T}=20^{\circ} \mathrm{C}, 40^{\circ} \mathrm{C}$, and $80^{\circ} \mathrm{C}$. Before running the production trajectories at such temperatures, the starting microstructures were equilibrated using the following strategy: $1 \times 10^{4}$ steps of steepest descent energy minimization were performed in order to relax conformational and structural tensions. Next, different consecutive rounds of MD runs were performed in order to equilibrate the density, temperature, and pressure. Specifically, all atoms of the system were submitted to $2 \mathrm{~ns}$ of steady heating until the target temperature was reached (i.e., $20^{\circ} \mathrm{C}, 40^{\circ} \mathrm{C}$, or $80^{\circ} \mathrm{C}$ ), $4 \mathrm{~ns}$ of $N V T$-MD at the desired temperature (thermal equilibration) followed by $10 \mathrm{~ns}$ of density relaxation (NPT-MD). Both temperature and pressure were controlled by the weak coupling method of the Berendsen thermobarostat, ${ }^{44}$ using a time constant for the heat bath coupling and a pressure relaxation time of 1 ps. After this, each NPT-MD production run at the desired temperature and $1 \mathrm{~atm}$ was $10 \mathrm{~ns}$ long. The numerical integration step was set to $1 \mathrm{fs}$ while the coordinates of the production run were saved every 2000 steps ( 2 ps interval).

\section{B. Determination of the glass transition}

Figure 3(a) displays the heating trace of P3TMA samples $(5 \mathrm{mg})$ at a heating rate of $1{ }^{\circ} \mathrm{C} / \mathrm{min}$. Before DSC measurement, samples were quenched from the melt state in 
(a)

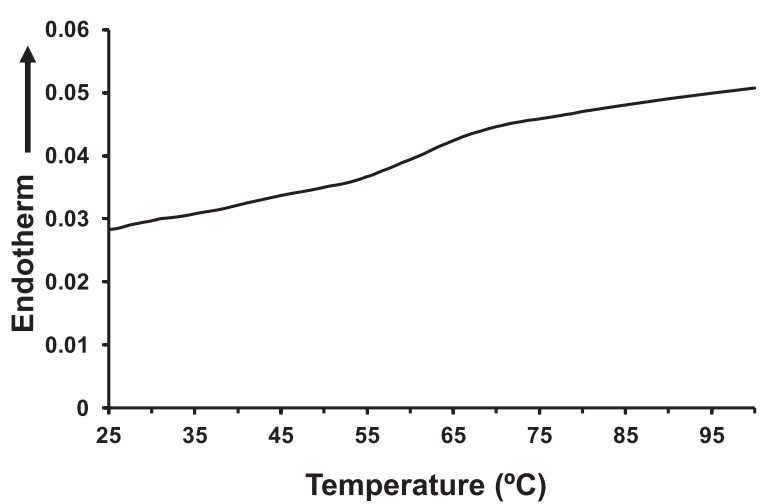

(b)

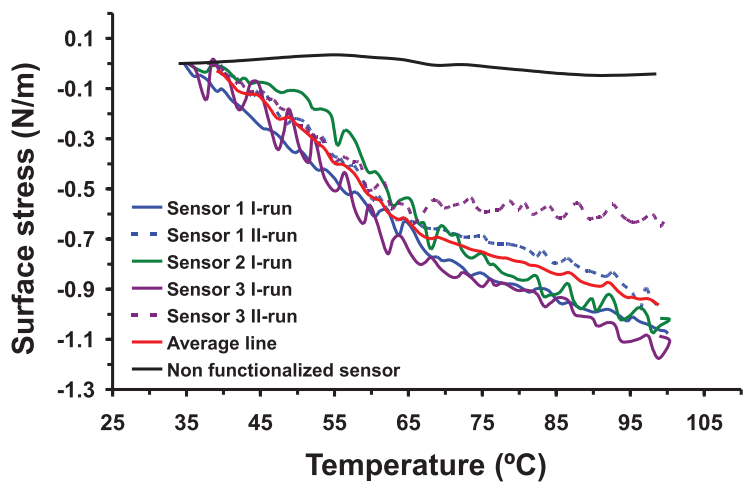

(c)

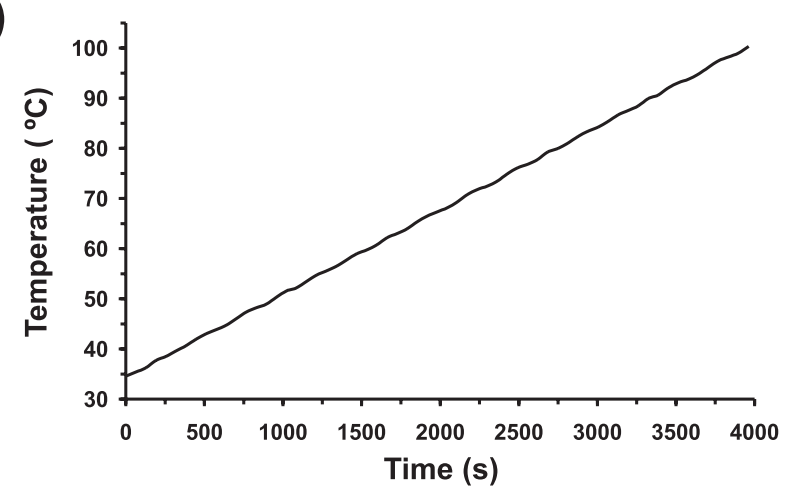

FIG. 3. (a) DSC heating trace $\left(1^{\circ} \mathrm{C} / \mathrm{min}\right)$ showing the glass transition associated with P3TMA bulk powder samples. (b) Deflection curve measured using microcantilever sensors for ultra-thin P3TMA samples. The graphic includes the first run for three sensors, the second run for two sensors, the average profile and a control sensor where no functionalization has been performed, (c) ramp of temperature.

order to increase the amorphous content and the glass transition signal. This is critical due to the low heating rate and the semicrystalline character of the material directly obtained from synthesis (melting point: $111.3^{\circ} \mathrm{C}$; X-ray diffraction peak at $1.17 \mathrm{~nm}$ ). The DSC heating trace shows a clear change on the base line allowing us to unambiguously determine the glass transition temperature at $\mathrm{T}_{\mathrm{g}}=61.8^{\circ} \mathrm{C}$.

On the other hand, P3TMA was solubilized in THF and deposited by spin-coating onto the surface of cantilever sensors for measurements. The thickness of the coating, which was determined by scratch AFM, was found to be $47 \mathrm{~nm}$. The densities measured for the bulk powder and the nanofilm samples, which were determined by the flotation method from
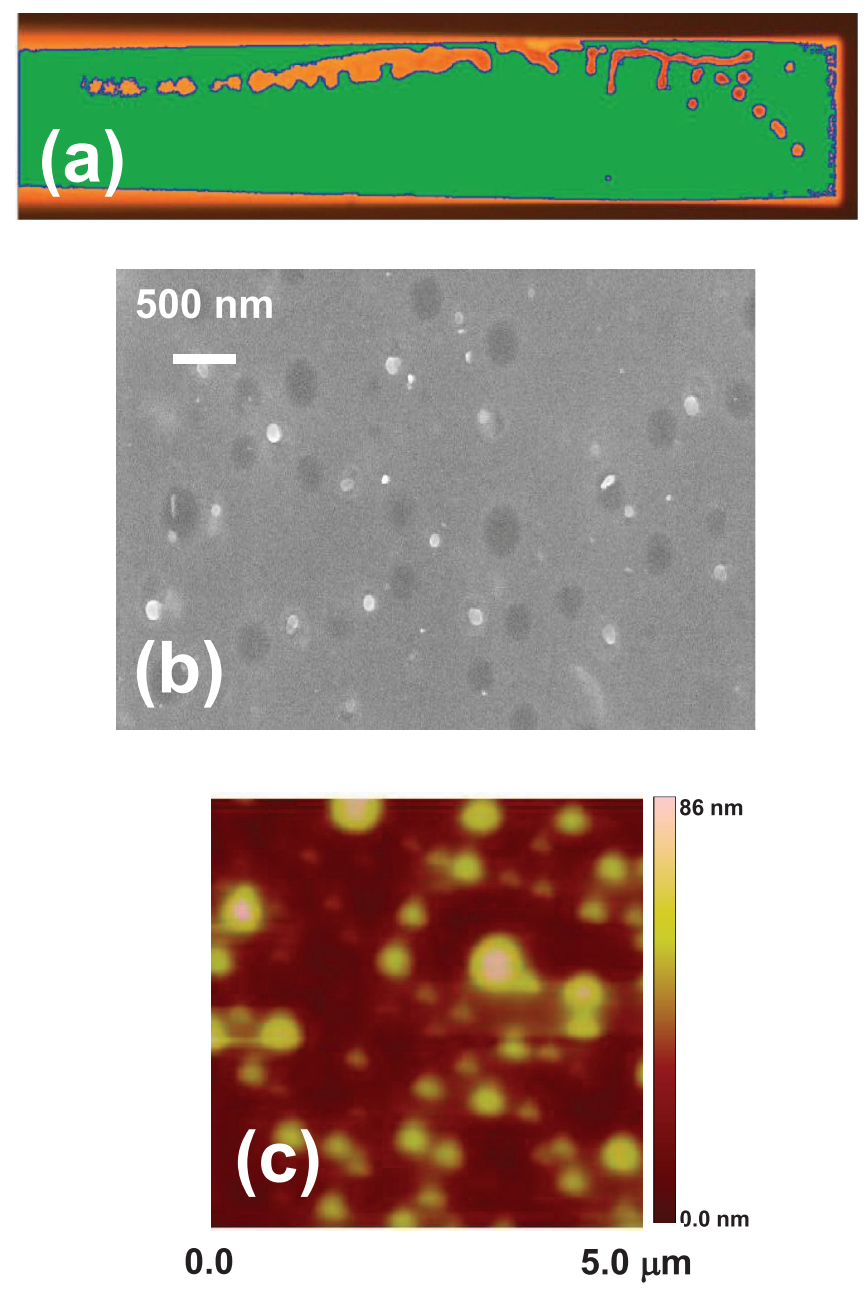

FIG. 4. (a) Optical image showing the surface of the microcantilever covered by P3TMA. AFM images of P3TMA: (b) bulk powder and (c) ultra-thin coating samples.

water+ethanol mixtures, were 0.976 and $1.000 \mathrm{~g} \mathrm{~cm}^{-3}$, respectively. Thus, the change from three mesoscopic dimensions to one nano-dimension combined with two macroscopic dimensional produces an increment of $2.5 \%$ in the density of P3TMA. This effect, which is expected to increase with the reduction of the nanomembrane thickness, should be attributed to the influence of the film-air interface in the molecular chains. Finally, the surface of the sensor covered by P3TMA was estimated to be around $\sim 10000 \mu \mathrm{m}^{2}$ in average (Figure 4(a)). According to these parameters, the mass of polymer deposited on each cantilever sensor is $\sim 10^{-13} \mathrm{~g}$, which represents a mass reduction of ten orders of magnitude with respect to conventional DSC measurements.

Figure 3(b) displays the deflection curves recorded using three different microcantilever sensors. As it can be seen, the three show a change in the slope at approximately the same temperature reflecting the reproducibility of the technique. Moreover, Figure 3(b) displays the second run recorded for two of the sensors, which evidence a variation in the slope of the profile just at the same position. A control sensor has been also included in Figure 3(b) to show the behavior of a nonfunctionalized cantilever. Figure 3(c) shows the ramp during the heating process (i.e., $1^{\circ} \mathrm{C} / \mathrm{min}$ ). The value of the glass 

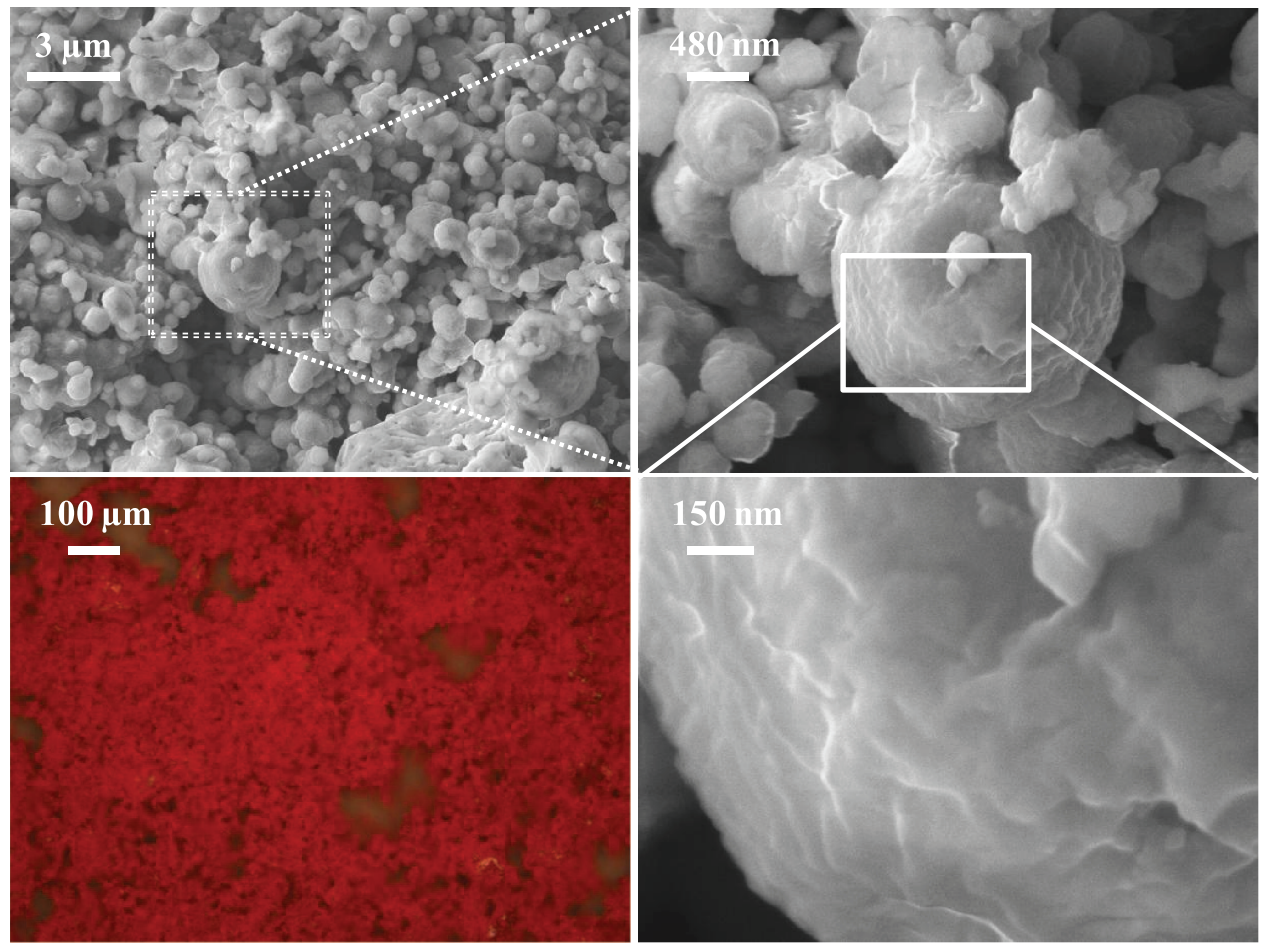

FIG. 5. Optical image $(20 \times$, bottom left) and SEM micrographs $(15 \mathrm{~K} \times$, top left; $60 \mathrm{~K} \times$, top right; and $220 \mathrm{~K} \times$ bottom right $)$ of P3TMA bulk powder samples.

transition of P3TMA at the nanometric scale, $\mathrm{T}_{\mathrm{g}}=67^{\circ} \mathrm{C}$, has been determined using the average curve (Figure 3(b)). This represents an increment of $5.2^{\circ} \mathrm{C}$ with respect to the bulk samples used for conventional DSC measurements at the same heating rate.

The effect of cantilever-polymer film interface on measurements of surface stress values has not been considered in this work. This is because such interface has been considered to behave ideally. More specifically, the molecular weight of P3TMA is high enough to transmit the stress along the contact points between the substrate and the polymer chains (i.e., formed crystals or entanglements), generating the force to bend of the cantilever. Above the percolation threshold, this behavior will be maintained even with few contact points. Indeed, the bend of the cantilevers with the temperature through biomaterial effect indicates by itself a good affinity between the polymer and the substrate. Accordingly, despite neglecting the interface effects, the stress transmitted by the cantilever allows to distinguish two regimes, below and above the $\mathrm{T}_{\mathrm{g}}$. Moreover, it should be mentioned that the surface stress versus temperature trend displayed in Figure 3(b) has been tested in more than 70 sensors, a very similar shape being obtained in all cases.

Powder sample used for DSC experiments and ultra-thin film deposited in the cantilever sensors present very different morphologies. Figure 5 displays optical and SEM images of powder samples, which present a granular morphology made of spherical particles with diameters ranging from $\sim 0.5$ to $\sim 2.0 \mu \mathrm{m}$. On the other hand, SEM micrographs (Figure 4(b)) and AFM images (Figure 4(c)) of the ultra-thin films evidence spherical nanoaggregates of small $(40-70 \mathrm{~nm})$ and medium (250-340 nm) size, bigger particles detaching from the polymer surface. The RMS roughness measured for the P3TMA film is $47 \mathrm{~nm}$. These morphological differences suggest that the $5.2^{\circ} \mathrm{C}$ increment in the $\mathrm{T}_{\mathrm{g}}$ produced by the reduction of the mass scale from $\sim 10^{-3} \mathrm{~g}$ to $\sim 10^{-13} \mathrm{~g}$ is due to a change in the microscopic organization of the polymer molecules. In order to investigate this feature at the atomistic level, MD simulations on model systems mimicking both the bulk and the ultra-thin coating have been carried out.

\section{Molecular dynamics simulations}

Analysis of the density (Table I) reveals significant differences between the BP and UTC models. The density predicted for the BP model varies less than $3 \%$ with the temperature, remaining within the $0.989-0.963 \mathrm{~g} \mathrm{~cm}^{-3}$ interval. These densities are fully consistent with the value obtained by flotation at room temperature for bulk powder samples (i.e., $0.976 \mathrm{~g} \mathrm{~cm}^{-3}$ ). In contrast, the densities calculated for the UTC model at $\mathrm{T}<\mathrm{T}_{\mathrm{g}}$ were $\sim 1.22 \mathrm{~g} \mathrm{~cm}^{-3}$, this value being significantly higher than that determined for the BP model. This feature is in qualitative agreement with experimental observations (i.e., the density of bulk samples was found to be

TABLE I. Average density and end-to-end distance for the BP and UTC models obtained from MD simulations at $\mathrm{T}=20^{\circ} \mathrm{C}, 40^{\circ} \mathrm{C}$, and $80^{\circ} \mathrm{C}$.

\begin{tabular}{llll}
\hline \hline & & Bulk powder & Ultra-thin coating \\
\hline Density $\left(\mathrm{g} \mathrm{cm}^{-3}\right)$ & $20^{\circ} \mathrm{C}$ & $0.989 \pm 0.005$ & $1.275 \pm 0.243$ \\
& $40^{\circ} \mathrm{C}$ & $0.982 \pm 0.007$ & $1.281 \pm 0.029$ \\
& $80^{\circ} \mathrm{C}$ & $0.963 \pm 0.005$ & $1.169 \pm 0.027$ \\
End-to-end distance $(\AA)$ & $20{ }^{\circ} \mathrm{C}$ & $27.86 \pm 0.09$ & $27.90 \pm 0.11$ \\
& $40^{\circ} \mathrm{C}$ & $27.80 \pm 0.10$ & $27.84 \pm 0.13$ \\
& $80^{\circ} \mathrm{C}$ & $26.78 \pm 0.10$ & $25.34 \pm 0.13$ \\
\hline \hline
\end{tabular}




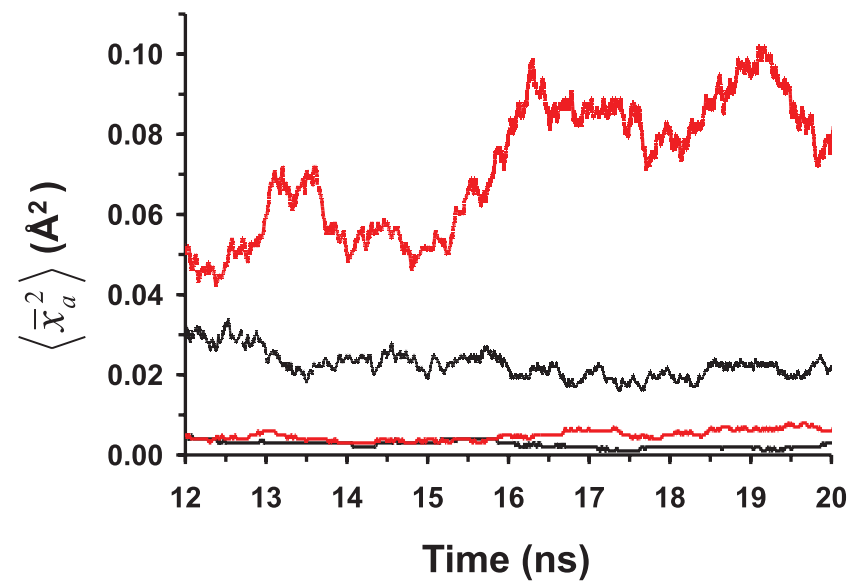

FIG. 6. Temporal evolution of the averaged atomic mean square displacement, $\left\langle\bar{x}_{a}^{2}\right\rangle$ for the BP (solid lines) and UTC (dashed lines) models at $\mathrm{T}=20^{\circ} \mathrm{C}$ and $80^{\circ} \mathrm{C}$ (black and red, respectively).

lower than that of nanometric film), even though the theoretical value is overestimated because of the nanodimensional simplicity of the model used for the simulations. Thus, the contribution of the interface effects in the UTC model is overvalued with respect to the nanofilm because the nanodimension is significantly smaller in the former than in the latter. Furthermore, the density predicted for the UTC model decreases $\sim 10 \%$ at $\mathrm{T}=80^{\circ} \mathrm{C}$ (i.e., $1.148 \mathrm{~g} \mathrm{~cm}^{-3}$ ). These results suggest not only some structural differences between the BP and UTC models but also some temperature-induced changes in the latter when $\mathrm{T}>\mathrm{T}_{\mathrm{g}}$. Differences between the two models are also reflected by the averaged atomic mean square displacement, $\left\langle\bar{x}_{a}^{2}\right\rangle$, which is represented in Figure 6 for the $10 \mathrm{~ns}$ of the production simulations at $\mathrm{T}=20^{\circ} \mathrm{C}$ and $80^{\circ} \mathrm{C}$. For the BP model, the values $\left\langle\bar{x}_{a}^{2}\right\rangle$ are small and similar at the two temperatures. In contrast, this property is one order of magnitude higher for the UTC model at $\mathrm{T}=20^{\circ} \mathrm{C}$, increasing significantly at $\mathrm{T}=80^{\circ} \mathrm{C}$.

Analysis of the inter-ring $\mathrm{S}-\mathrm{C}^{\alpha}-\mathrm{C}^{\alpha}-\mathrm{S}\left(\chi_{1}\right)$ and side group $\mathrm{C}^{\beta}-\mathrm{C}\left(\mathrm{H}_{2}\right)-\mathrm{C}(=\mathrm{O})-\mathrm{O}\left(\chi_{2}\right)$ dihedral angles reveals that the differences between the BP and UTC models essentially refer to $\chi_{1}$. This is reflected in Table II, which summarizes the distribution of $\chi_{1}$ and $\chi_{2}$ at $\mathrm{T}=20^{\circ} \mathrm{C}$ and $80^{\circ} \mathrm{C}$. As expected, the most populated conformation at $\mathrm{T}=20^{\circ} \mathrm{C}$ for $\chi_{1}$ is the anti one, independent of the model (i.e., $84.9 \%$ and $85.1 \%$ for BP and UTC, respectively), even though the population of the syn arrangement is relatively high (i.e., $10.9 \%$ and $9.0 \%$ for BP and UTC, respectively). These results are fully consistent with the rotational profile predicted by quantum mechanical methods for the inter-ring dihedral angle, ${ }^{45-49}$ which indicated that the syn conformation is severely destabilized when adjacent substituted thiophene rings show a head-head linkage but energetically accessible for head-tail and tail-tail linkages. From an electronic point of view, the distortion of the interring dihedral angle from anti to syn destroys the extent of the $\pi$-conjugation along the polymer chain. Results obtained from the simulations at $\mathrm{T}=40^{\circ} \mathrm{C}$ (not shown) were practically the same than those at $\mathrm{T}=20^{\circ} \mathrm{C}$. The population of the anti and syn conformations decreases and increases, respectively, upon heating to $\mathrm{T}=80^{\circ} \mathrm{C}$, even though this change is
TABLE II. Distribution of the dihedral angles $\chi_{1}$ and $\chi_{2}$ for the BP and UTC models as obtained from MD simulations at $\mathrm{T}=20$ and $80^{\circ} \mathrm{C}$.

\begin{tabular}{|c|c|c|c|c|c|c|c|c|}
\hline & \multicolumn{4}{|c|}{ Bulk powder } & \multicolumn{4}{|c|}{ Ultra-thin coating } \\
\hline & \multicolumn{2}{|c|}{$\mathrm{T}=20^{\circ} \mathrm{C}$} & \multicolumn{2}{|c|}{$\mathrm{T}=80^{\circ} \mathrm{C}$} & \multicolumn{2}{|c|}{$\mathrm{T}=20^{\circ} \mathrm{C}$} & \multicolumn{2}{|c|}{$\mathrm{T}=80^{\circ} \mathrm{C}$} \\
\hline & $\chi_{1}$ & $\chi_{2}$ & $\chi_{1}$ & $\chi_{2}$ & $\chi_{1}$ & $\chi_{2}$ & $\chi_{1}$ & $\chi_{2}$ \\
\hline $30^{\circ} / 90^{\circ}$ & 0.5 & 13.0 & 0.9 & 11.0 & 1.0 & 14.7 & 0.9 & 7.6 \\
\hline $90^{\circ} / 150^{\circ}$ & 1.4 & 23.3 & 1.3 & 24.3 & 1.8 & 23.3 & 1.6 & 30.4 \\
\hline $150^{\circ} /-150^{\circ}$ & 84.9 & 25.1 & 76.9 & 26.6 & 85.1 & 24.1 & 61.1 & 40.7 \\
\hline$-150^{\circ} /-90^{\circ}$ & 1.5 & 25.3 & 2.2 & 28.2 & 2.3 & 22.6 & 1.7 & 22.9 \\
\hline$-90^{\circ} /-30^{\circ}$ & 0.8 & 12.4 & 0.9 & 8.8 & 0.8 & 14.1 & 0.5 & 8.3 \\
\hline$-30^{\circ} / 30^{\circ}$ & 10.9 & 0.9 & 17.8 & 1.1 & 9.0 & 1.2 & 34.2 & 1.1 \\
\hline
\end{tabular}

significantly more important for the UTC model than for the BP one (i.e., the population of $s y n$ at $\mathrm{T}=80^{\circ} \mathrm{C}$ is $34.2 \%$ and $17.8 \%$ for the BP and UTC model, respectively). Despite such conformational change, the influence of the temperature in the average end-to-end distance, which was calculated using the backbone atoms of the thiophene rings, is relatively small (Table I). Thus, the value obtained for the BP and UTC models decreases 1.08 and $2.56 \AA$, respectively, when the temperature increases from $20^{\circ} \mathrm{C}$ to $80^{\circ} \mathrm{C}$.

Independent of the model, the dihedral angle $\chi_{2}$ is able to adopt a wide range of values at $\mathrm{T}=20^{\circ} \mathrm{C}$ and $40^{\circ} \mathrm{C}$, as it is indicated in Table II. Thus, populations are higher than $12 \%$ for all the intervals with exception of that comprised between $-30^{\circ}$ and $30^{\circ}$, which is sterically precluded. The conformational behavior of the two models at $\mathrm{T}=80{ }^{\circ} \mathrm{C}$ is different (Table II). For the BP model, the populations of the intervals comprised between $90^{\circ}$ and $-90^{\circ}$ increase slightly with respect to $\mathrm{T}=20^{\circ} \mathrm{C}$ (i.e., from $23.3 \%-25.3 \%$ to $24.3 \%-$ $28.2 \%$ when the temperature increases from $20^{\circ} \mathrm{C}$ to $80^{\circ} \mathrm{C}$ ). In contrast, for the UTC model the population of the interval comprised between $150^{\circ}$ and $-150^{\circ}$ increases to $40.7 \%$ at $\mathrm{T}=80^{\circ} \mathrm{C}$, evidencing that the conformational variability of the latter model is higher than that of the BP one.

MD results indicate that the film-air interface, which has been induced in the UTC model by eliminating the periodic boundary conditions along the z-axis, affects the packing and distribution of the polymer chains. This interface breaks the isotropy of the BP model, in which periodic boundary conditions were applied in the three axes. The phase rupture imposed in the UTC model affects the density and the conformation of the molecular chains.

\section{CONCLUSIONS}

A sensing technology based on microcantilevers has been developed to study the thermal transitions of polymers. The technology has been used to investigate the glass transition of a polythiophene with different applications in nanotechnology. The $\mathrm{T}_{\mathrm{g}}$ determined for ultra-thin samples of $\sim 10^{-13} \mathrm{~g}$ is $5.2{ }^{\circ} \mathrm{C}$ higher than that obtained for $5 \mathrm{mg}$ of bulk powder using conventional differential scanning calorimetry. SEM images reflect important morphological differences between the bulk powder and ultra-thin samples, suggesting a different microscopic organization of the polymer molecules. Atomistic MD 
simulations on models periodic boundary conditions applied along three and two directions have been used to examine such differences. Calculations indicate that the film-air interface affects not only the density, which is in agreement with experimental observations, but also the conformation of the polymer molecules. The overall of these results reflect how important is the development of new technologies to investigate the thermal transitions in samples similar to those used in nanotechnology.

\section{ACKNOWLEDGMENTS}

This work has been supported by MICINN and FEDER (Grant Nos. MAT2009-09138 and MAT2009-11513), and by the Generalitat de Catalunya (Grant Nos. 2009-SGR-925, 2009-SGR-1208, and XRQTC). The authors are indebted to the "Centre de Supercomputació de Catalunya" (CESCA) for the computational resources provided. M.M.P.-M. thanks financial support through a FPI-UPC grant. Support for the research of C.A. was received through the prize "ICREA Academia" for excellence in research funded by the Generalitat de Catalunya.

${ }^{1}$ D. R. Queen and F. Hellman, Rev. Sci. Instrum. 80, 063901 (2009).

${ }^{2}$ W. Lee, W. Fon, B. W. Axelrod, and M. L. Roukes, Proc. Natl. Acad. Sci. U.S.A. 106, 15225 (2009).

${ }^{3}$ S. L. Lai, J. Y. Guo, V. Petrova, G. Ramanath, and L. H. Allen, Phys. Rev. Lett. 77, 99 (1996).

${ }^{4}$ F. Yi and D. A. La Van, Nanomed. Nanobiotechnol. 4, 31 (2012).

${ }^{5}$ P. Swaminathan, B. G. Burke, A. E. Holness, B. Wilthan, L. Hanssen, T. P. Weihs, and D. A. LaVan, Thermochim. Acta 522, 60 (2011).

${ }^{6}$ R. L. Greene, C. N. King, and R. B. Zubeck, Phys. Rev. B 6, 3297 (1972).

${ }^{7}$ J. L. Garden, H. Guillou, A. F. Lopeandia, J. Richard, J. S. Heron, G. M. Souche, F. R. Ong, B. Vianay, and O. Bourgeois, Thermochim. Acta 492, 16 (2009).

${ }^{8}$ J. Lerchner, T. Maskow, and G. Wolf, Chem. Eng. Process. 47, 991 (2008).

${ }^{9}$ R. K. Kummamuru, L. de la Rama, L. Hu, M. D. Vaudin, M. Y. Efremov, M. L. Green, D. A. LaVan, and L. H. Allen, Appl. Phys. Lett. 95, 181911 (2009).

${ }^{10}$ L. P. Cook, R. E. Cavicchi, M. L. Green, C. B. Montgomery, and W. F. Egelhoff, AIP Conf. Proc. 931, 151 (2007).

${ }^{11}$ L. Wang, B. Wang, and Q. Lin, Sens. Actuators B 134, 953 (2008).

${ }^{12}$ J. Lerchner, R. Kirchner, J. Seidel, D. Waehlisch, and G. Wolf, Thermochim. Acta 415, 27 (2004)

${ }^{13}$ E. Meyer, J. K. Gimzewski, Ch. Gerber, and R. R. Schlittler, in The Ultimate Limits of Fabrication and Measurement, edited by M. E. Welland and J. K. Gimzewski (Kluwer, Dordrecht, 1995), pp. 89-95.

${ }^{14}$ C. E. Borroni-Bird, N. Al-Sarraf, S. Andersoon, and D. A. King, Chem. Phys. Lett. 183, 516 (1991).

${ }^{15}$ J. R. Barnes, R. J. Stephenson, M. E. Welland, Ch. Gerber, and J. K. Gimzewski, Nature (London) 372, 79 (1994).

${ }^{16}$ N. Jung, H. Seo, D. Lee, C. Y. Ryu, and S. Jeon, Macromolecules 41, 6873 (2008).

${ }^{17}$ A. Mader, K. Gruber, R. Castelli, B. A. Hermann, P. H. Seeberger, J. O. Radler, and M. Leisner, Nano Lett. 12, 420 (2012).
${ }^{18}$ K. Gruber, T. Horlarcher, R. Castelli, A. Mader, P. H. Seeberger, and B. A. Hermann, ACS Nano 5, 3670 (2011).

${ }^{19}$ A. Anne, C. Demaille, and C. Goyer, ACS Nano 3, 819 (2009).

${ }^{20}$ G. Yoshikawa, T. Akiyama, S. Gautsch, P. Vettiger, and H. Roher, Nano Lett. 11, 1044 (2011).

${ }^{21}$ L. Lechuga Gómez, V. Álvarez-Sanchéz, and F. J. T. de Miguel, U.S. patent 7,646,494 B2 (12 January 2007).

${ }^{22}$ F. J. Tamayo De Miguel, J. Mertens, and M. Calleja-Gómez, U.S. patent 7,978,344 B2 (12 July 2011).

${ }^{23}$ N. F. Martinez, P. M. Kosaka, J. Tamayo, J. Ramirez, O. Ahumada, J. Mertens, T. D. Hien, C. V. Rijn, and M. Calleja, Rev. Sci. Instrum. 81, 125109 (2010).

${ }^{24}$ J. Tamayo, V. Pini, P. M. Kosaka, N. F. Martinez, O. Ahumada, and M. Calleja, Nanotechnology 23, 315501 (2012).

${ }^{25} \mathrm{R}$. Berger, Ch. Gerber, J. K. Ginzewski, E. Meyer, and H. J. Güntherodt, Appl. Phys. Lett. 69, 40 (1996).

${ }^{26}$ W.-H. Chu, M. Mehregany, and R. L. Mullen, J. Micromech. Microeng. 3, 4 (1993).

${ }^{27}$ D. Ramos, J. Mertens, M. Calleja, and J. Tamayo, Sensors 7, 1757 (2007).

${ }^{28}$ M. Yun, N. Jung, C. Yim, and S. Jeon, Polymer 52, 4136 (2011).

${ }^{29}$ J. R. Barnes, R. J. Stephenson, C. N. Woodburn, S. J. O'Shea, M. E. Welland, T. Rayment, J. K. Gimzewski, and Ch. Gerber, Rev. Sci. Instrum. 65, 3793 (1994)

${ }^{30}$ K. Gimzewski, Ch. Gerber, E. Meyer, and R. R. Schlittler, Chem. Phys. Lett. 217, 589 (1994).

${ }^{31}$ E. Armelin, A. L. Gomes, M. M. Pérez-Madrigal, J. Puiggalí, L. Franco, L. J. del Valle, A. Rodíguez-Galán, J. S. de C. Campos, N. Ferrer-Anglada, and C. Alemán, J. Mater. Chem. 22, 585 (2012).

${ }^{32}$ M. M. Pérez-Madrigal, E. Armelin, L. J. del Valle, F. Estrany, and C. Alemán, Polym. Chem. 3, 979 (2012).

${ }^{33}$ M. Martí, G. Fabregat, D. S. Azambuja, C. Alemán, and E. Armelin, Prog. Org. Coat. 73, 321 (2012).

${ }^{34}$ E. Armelin, C. Alemán, J. I. Iribarren, F. Liesa, and F. Estrany, Patent Cooperation Treaty PCT/ES2010070820 (21 June 2012).

${ }^{35}$ E. Armelin, C. Alemán, J. I. Iribarren, F. Liesa, and F. Estrany, Patent Cooperation Treaty U.S. application 13/138925 (10 September 2012).

${ }^{36}$ B. Kim, L. Chen, J. Gong, and Y. Osada, Macromolecules 32, 3964 (1999).

${ }^{37}$ A. L. Gomes, J. Casanovas, O. Bertran, J. S. de C. Campos, E. Armelin, and C. Alemán, J. Polym. Res. 18, 1509 (2011).

${ }^{38}$ J. C. Phillips, R. Braun, W. Wang, J. Gumbart, E. Tajkhorshid, E. Villa, C. Chipot, R. D. Skeel, L. Kale, and K. Schulten, J. Comput. Chem. 26, 1781 (2005).

${ }^{39}$ W. D. Cornell, P. Cieplak, C. I. Bayly, I. R. Gould, K. M. Merz, D. M. Ferguson, D. C. Spellmeyer, T. Fox, J. W. Caldwell, and P. A. Kollman, J. Am. Chem. Soc. 117, 5179 (1995).

${ }^{40}$ J. Wang, R. M. Wolf, J. W. Caldwell, and D. A. Case, J. Comput. Chem. 15, 1157 (2004).

${ }^{41}$ P. Cieplak, W. Cornell, C. I. Bayly, and P. A. Kollman, J. Comput. Chem. 16, 1357 (1995).

${ }^{42}$ J. P. Ryckaert, G. Ciccotti, and H. J. C. Berendsen, J. Comput. Phys. 23, 327 (1977).

${ }^{43}$ D. Curcó and C. Alemán, J. Comput. Chem. 28, 1743 (2007).

${ }^{44}$ H. J. C. Berendsen, J. P. M. Postma, W. F. van Gunsteren, A. Dinola, and J. R. Haak, J. Chem. Phys. 81, 3684 (1984).

${ }^{45}$ C. Alemán and L. Julia, J. Phys. Chem. 100, 1524 (1996).

${ }^{46}$ C. Alemán, V. M. Domingo, L. Fajarí, L. Juliá, and A. Karpfen, J. Org. Chem. 63, 1041 (1998).

${ }^{47}$ O. Bertran, E. Armelin, J. Torras, F. Estrany, M. Codina, and C. Alemán, Polymer 49, 1972 (2008).

${ }^{48}$ E. Armelin, O. Betran, F. Estrany, R. Salvatella, and C. Alemán, Eur. Polym. J. 45, 2211 (2009)

${ }^{49}$ O. Bertran, P. Pfeiffer, J. Torras, E. Armelin, F. Estrany, and C. Alemán, Polymer 48, 6955 (2007). 